\title{
EUV-dependence of Venusian dayside ionopause altitude: VEX and PVO observations
}

\author{
QianQian Han ${ }^{1,2,3}$, Markus Fraenz ${ }^{4}$, Yong Wei ${ }^{1,3,5 *}$, Eduard Dubinin ${ }^{4}$, Jun Cui ${ }^{6,7}$, LiHui Chai ${ }^{1,3}$, ZhaoJin Rong ${ }^{1,3}$, \\ WeiXing Wan ${ }^{1,3}$, and Yoshifumi Futaana ${ }^{8}$ \\ ${ }^{1}$ Key Laboratory of Earth and Planetary Physics, Institute of Geology and Geophysics, Chinese Academy of Sciences, Beijing 100029, China; \\ ${ }^{2}$ Beijing National Observatory of Space Environment, Institute of Geology and Geophysics, Chinese Academy of Sciences, Beijing 100049, China; \\ ${ }^{3}$ College of Earth and Planetary Sciences, University of Chinese Academy of Sciences, Beijing 100049, China; \\ ${ }^{4}$ Max-Planck-Institute for Solar System Research, Goettingen, DE 37077, Germany; \\ 5 Institutions of Earth Science, Chinese Academy of Sciences, Beijing 100029, China; \\ ${ }^{6}$ School of Atmospheric Sciences, Sun Yat-Sen University, Zhuhai Guangdong 519082, China; \\ ${ }^{7}$ Key Laboratory of Lunar and Deep Space Exploration, National Astronomical Observatories, Chinese Academy of Sciences, Beijing 100012, China; \\ ${ }^{8}$ Swedish Institute of Space Physics, Kiruna, SE 98128, Sweden
}

\section{Key Points:}

- This is the first attempt to combine PVO and VEX data to investigate how ionopause altitude varies with levels of solar activity

- We have derived a relationship between solar EUV level and the dayside ionopause altitude

- We find that dayside ionopause altitude increases as solar EUV level increases, which is consistent with theoretical expectations

Citation: Han, Q. Q., Fraenz, M., Wei, Y., Dubinin, E., Cui, J. Chai, L. H., Rong, Z. J., Wan, W. X., and Futaana, Y. (2020). EUV-dependence of Venusian dayside ionopause altitude: VEX and PVO observations. Earth Planet. Phys., 4(1), 73-81. http://doi.org/10.26464/epp2020011

\begin{abstract}
The Venusian dayside ionosphere, similar to other planetary ionospheres, is produced primarily by ionization of its neutral upper atmosphere due to solar extreme ultraviolet (EUV) radiation. It has become clear that the expansion of the ionosphere may be strongly controlled by the EUV level, as exhibited in data collected by the Pioneer Venus Orbiter (PVO) during one solar cycle (1978-1992). However, the EUV-dependence of the Venusian dayside ionopause altitude, which defines the outer boundary of the ionosphere, remains obscure because the PVO crossed the dayside ionopause only during the solar maximum; its periapsis lifted too high during the solar minimum. Recently, during the period 2006-2014, which included the longest and quietest solar minimum of the past several decades, Venus Express (VEX) provided measurements of the photoelectron boundary (PEB) over the northern high-latitude region. Since the photoelectron boundary is closely related to the ionopause, we have an opportunity to analyze the EUV effect on the dayside ionopause by combining PVO and VEX observations. We have evaluated and then reduced the orbit bias effect in data from both PVO and VEX, and then used the results to derive a relationship between solar EUV level and the dayside ionopause altitude. We find that the dayside ionopause altitude increases as the solar EUV level increases, which is consistent with theoretical expectations.
\end{abstract}

Keywords: Venus; ionopause; ionosphere; solar activity

\section{Introduction}

Venus's ionosphere is similar to Earth's ionosphere in many aspects, but it has an ionopause created by direct interaction with solar wind. Since Venus lacks an intrinsic magnetic field, the planet's ionosphere interacts directly with the shocked solar wind plasma. The ionospheric plasma is a very good electrical conductor; when interacting with the solar wind, the ionosphere acts at large sale like a highly conducting spheroid. It stands off the solar wind, and an ionopause is formed at the altitude where the iono-

Correspondence to: Y. Wei, weiy@mail.iggcas.ac.cn

Received 20 SEP 2019; Accepted 11 DEC 2019.

Accepted article online 09 JAN 2020.

(C)2020 by Earth and Planetary Physics. sphere's internal pressure is approximately balanced by external pressure (Luhmann, 1986). The external pressure includes the solar wind's dynamic pressure $\rho v^{2}$, thermal pressure $n k T$, and magnetic pressure $B^{2} / 2 \mu_{0}$. However, compared to its dynamic pressure, the thermal and magnetic pressure components of the solar wind can be neglected, except near the terminator, where the dynamic pressure's normal component goes to zero; in the terminator region, the solar wind's thermal and magnetic pressure contributions must be considered. The internal pressure of the ionosphere is its thermal pressure $n k T$, where $T=T_{\mathrm{e}}+T_{\mathrm{i}} ; T_{\mathrm{e}}$ is the electron temperature and $T_{\mathrm{i}}$ is the ion temperature. The magnetic pressure inside the ionopause also contributes to the internal pressure, becoming important when the ionosphere is significantly magnetized. When the solar wind approaches Venus, its flow is shocked and diverted around the Venusian ionosphere above 
the ionopause (Brace and Kliore, 1991; Luhmann, 1986).

Theoretically, the altitude of the ionopause is closely related to the solar extreme ultraviolet (EUV) level. The dayside ionosphere of Venus is created primarily by ionization of its neutral atmosphere. Because the ionosphere is photochemically controlled, its principal dayside layer behaves like a simple Chapman layer. In the photochemical equilibrium region for the case of a single major ion, the ion density $n_{\mathrm{i}}$ and electron density $n_{\mathrm{e}}$ should be proportional to $\sqrt{P / a}$, where $a$ is the effective value of the recombination coefficient and $P$ is the total ion production rate. The response of the major ion density profile to changes in solar flux can be predicted by Chapman theory. The ion production increases as the solar ionizing flux outside the atmosphere increases. When the EUV increases, the ionospheric thermal pressure $n k T$ increases as well, causing the altitude at which the ionospheric pressure balances the external pressure to increase. In other words, the altitude of the dayside ionopause varies with the strength of the solar EUV (Fox and Kliore, 1997).
Most of our knowledge about the Venusian ionopause comes from Pioneer Venus Orbiter (PVO) observations (reviewed by Brace and Kliore (1991)). The PVO operated for 14 years (1978-1992), from the maximum of solar cycle 21 to the maximum of solar cycle 22, and gave us a wealth of data for one entire solar cycle (see Figure 1a). From 1979 to 1980, the PVO frequently crossed the ionosphere, recording the ionopause on most orbits. However, the PVO's periapsis started to rise slowly after the summer of 1980 (see Figure 1b). By April 1981 the periapsis was above the average altitude of the dayside ionopause, so the PVO encountered the ionosphere only in the terminator regions and on the nightside. Only after 1992 when the periapsis returned to low enough altitudes, was the dayside ionopause again recorded. From 1981 to 1992, which included the solar minimum, the PVO rarely crossed the dayside ionopause - it did so only when the ionosphere was extremely expanded, as a consequence of tenuous solar wind rather than low solar EUV levels (Russell et al., 1993). Therefore, it is not possible to use PVO data to analyze the EUV effect on the dayside ionopause altitude over a whole solar

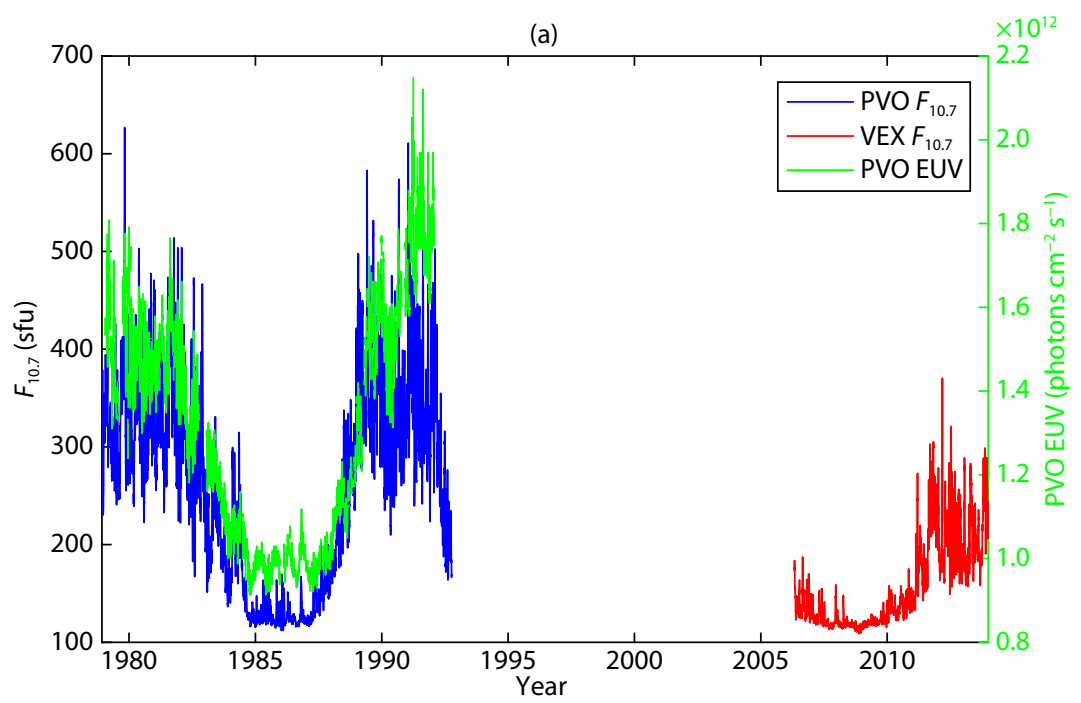

(b)

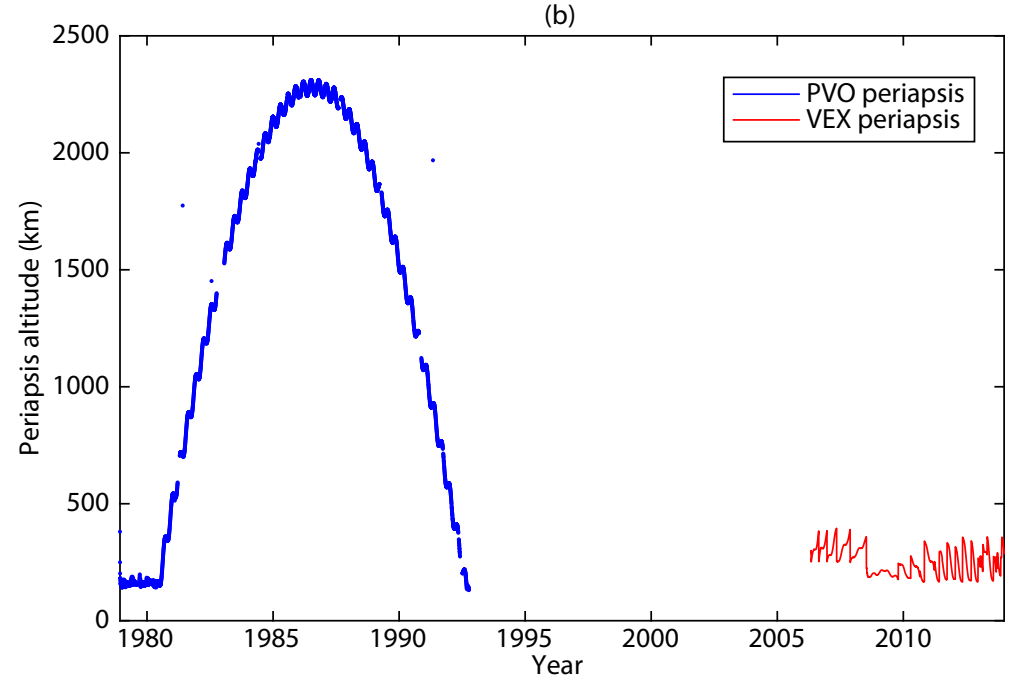

Figure 1. Comparison of the $F_{10.7}$ and periapsis of PVO and VEX. (a) The $F_{10.7}$ extrapolate from Earth to Venus. The blue and red curve represent the extrapolated $F_{10.7}$ during operation period of PVO and VEX, respectively. The green curve represents the EUV measured by PVO. The extrapolated $F_{10.7}$ during the PVO period is consistent well with the EUV measured by PVO. (b) The altitude of PVO and VEX periapsis. 
cycle.

Fortunately, Venus Express (VEX) had a low periapsis during its mission period (2006-2014), which included an unusually long solar minimum. VEX had an elliptic polar orbit and a 24-hour period, similar to PVO, and its periapsis was always above the Venus north pole. Since its periapsis altitude was maintained at $160-$ $400 \mathrm{~km}$ (see Figure 1b), on most orbits VEX crossed the dayside ionopause either inbound or outbound. Though VEX carried no onboard Langmuir probe, it had been proposed to use VEX measurements of the photoelectron distribution to identify the outer boundary of ionosphere, which could be a proxy of the ionopause, and many new features were disclosed with this method (Martinecz et al., 2008, 2009; Angsmann et al., 2011; Han X et al., 2014; Vech et al., 2015), which we, too, will follow (introduced in details in Section 2.1) to construct an ionopause database. Combining PVO and VEX data, we are then able to investigate the EUV effect on the ionopause over an entire solar cycle.

\section{Observations}

\subsection{Datasets}

Physically the term ionopause describes an extended altitude range over which the ionospheric plasma density falls off steeply, but to investigate the dependence of this altitude range on external parameters one needs to define a specific ionopause altitude. Several different definitions of the ionopause have been offered, depending on observations from different instruments. The different earlier datasets used to describe the Venusian ionopause indeed show similar global configurations (Phillips et al., 1988), and thus any of these can be used to investigate the response of the Venusian ionosphere to solar wind and/or EUV level. In our work, the ionopause datasets from PVO and VEX also come from different types of instrument.

The PVO dayside ionopause database (874 data points) is from the Orbiter Electron Temperature Probe (OETP). The OETP uses two cylindrical Langmuir probes (axial and radial) to measure ionospheric electron density $\left(n_{\mathrm{e}}\right)$, electron temperature $\left(T_{\mathrm{e}}\right)$, and ion density $\left(n_{\mathrm{i}}\right)$ (Krehbiel et al., 1980). It can detect ionospheric plasma when the spacecraft is in the ionosphere. The ionopause is defined at the point where $n_{\mathrm{e}}$ crosses through $100 \mathrm{~cm}^{-3}$ in a steep gradient; PVO data thus identifies the ionopause as the point where $n_{\mathrm{e}}$ first rises above the instrumental background density (Brace et al., 1980). When irregularities or waviness in the ionopause produce multiple ionopause crossings, the outer-most crossing is the only one selected. This database was built up by the PVO/OETP team before 1993 and now can be accessed through the Planetary Data System (PDS) website (https://pdsppi.igpp.ucla.edu/).

The VEX dayside ionopause database (1764 data points) is obtained from the plasma instrument Analyzer of Space Plasma and Energetic Atoms (ASPERA-4) (Barabash et al., 2007). Unlike PVO, VEX did not carry Langmuir probes to measure the ionospheric cold plasma. However, the Electron Spectrometer (ELS) in the ASPERA-4 package measures photoelectrons produced in the ionosphere. ELS is designed to detect electrons within the energy range from $1 \mathrm{eV}$ to $20 \mathrm{keV}$ with a time resolution of $4 \mathrm{~s}$, but due to spacecraft charging the real lower limit of the energy range is 5 $\mathrm{eV}$, making it impossible to detect the ionospheric cold plasma. The ELS frequently observed specific photoelectron lines in the energy spectrum between 18 and $25 \mathrm{eV}$ (real value 23 to $30 \mathrm{eV}$, taking into account the $-5 \mathrm{~V}$ spacecraft potential), indicating local production from $\mathrm{O}$ or $\mathrm{CO}_{2}$ atoms (Coates et al., 2008). Since these photoelectrons can propagate along magnetic field lines, the ionopause altitude should not be higher than the location at which a strong flux gradient of these photoelectrons occurs. Therefore, such a photoelectron boundary (PEB) may be used as an indicator of the ionopause altitude. Following this idea, a VEX dayside ionopause dataset can be built up by identifying the PEB (Martinecz et al., 2008, 2009; Angsmann et al., 2011). On the other hand, Han X et al. (2014) have shown that at Mars the PEB is observed at an altitude which is about $200 \mathrm{~km}$ higher than the location of the steepest cold electron gradient. Furthermore, Han QQ et al. (2019) have also investigated the relationship between the PEB and the steep electron density gradient. Their results show that, although on average the PEBs are located at higher altitudes, when observed on the same orbit the two boundaries are nearly collocated.

The PVO's solar EUV measurements are based on photoelectron emission from the onboard Langmuir probe; daily solar EUV values are based on the photoemission current $i_{\text {pe }}$ from the radial probe, which is proportional to the intensity of the ionizing component of the solar radiation. Thus the total solar EUV flux ( $V_{\mathrm{EUV}}$ ) can be derived from the daily $i_{\text {pe }}$ measurements using the relations $V_{\mathrm{EUV}}=1.53 \times 10^{11} i_{\mathrm{pe}}$ (photons $\left.\mathrm{cm}^{-2} \mathrm{~s}^{-1}\right)$, where $i_{\mathrm{pe}}$ is in units of $10^{-9}$ amperes (Brace et al., 1988). However, because VEX did not carry a UV instrument, we must use the $F_{10.7}$ extrapolated from Earth to Venus (Mitchell et al., 2001). Therfore, for consistency we use extrapolated $F_{10.7}$ values for both PVO and VEX, comparing extrapolated values during the PVO mission period with the EUV flux measured by PVO (Figure 1a). It is obvious that the extrapolated $F_{10.7}$ (blue curve) is quite consistent with the EUV flux measured by PVO (green curve). The time period of the VEX ionopause crossings used here included almost one complete solar cycle. However, the mean flux of $10.7 \mathrm{~cm}$ solar radiation during the VEX interval was lower than that during the PVO period (Figure 1a). Figure $2 a$ and Figure $2 b$, show the distributions of PVO and VEX dayside ionopause observations as a function of time, altitude, and solar zenith angle (SZA), respectively. The horizontal axis is time in years and vertical axis is the solar zenith angle in degrees; the color represents the altitude of the dayside ionopause. For the PVO dayside ionopause, the altitude is higher for larger SZA. It is obvious that a large data gap exists for PVO during 1982-1990, due to its raised orbit (Figure 1b). On the other hand, since VEX operated over almost one complete solar cycle, its data can fill the solar minimum data gap left by the PVO. Combining the PVO and VEX data sets in this way allows us to analyze the EUV effect on the dayside ionopause altitude over an entire solar cycle. It is clear that the averaged altitude of the dayside ionopause measured by VEX is lower than observed by PVO (Figure 2c). This may be attributed to the different mean EUV level, as is to be expected (Fox and Kliore, 1997). Before evaluating the relationship between VEX and PVO observations, we should first analyze and try to reduce orbit biases of both the PVO and VEX, in order to extract from these 


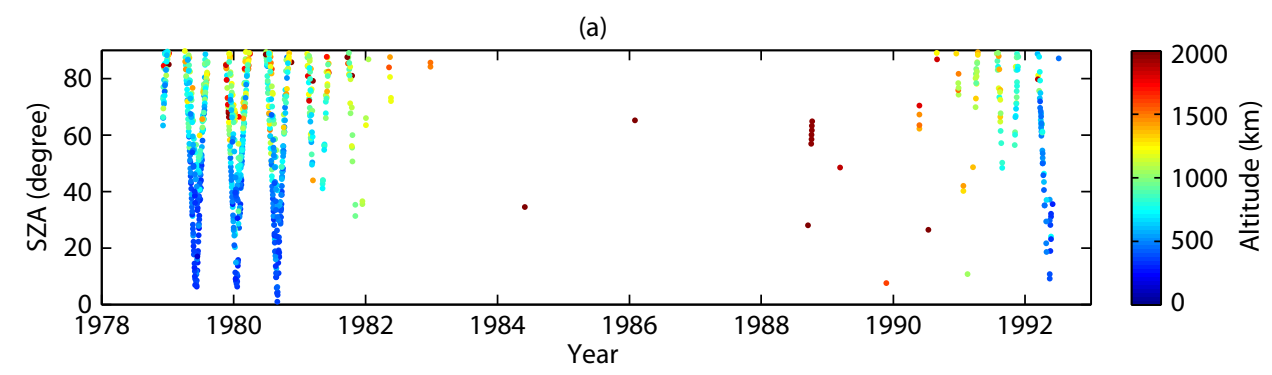

(b)

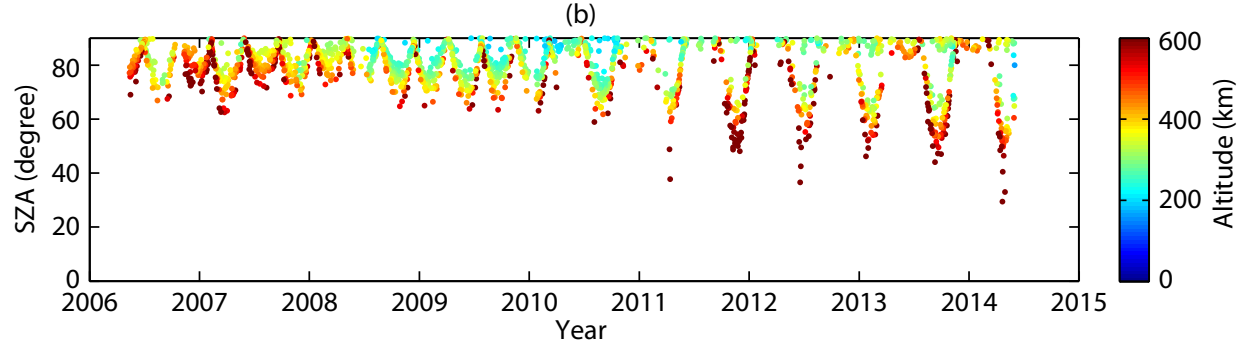

(c)

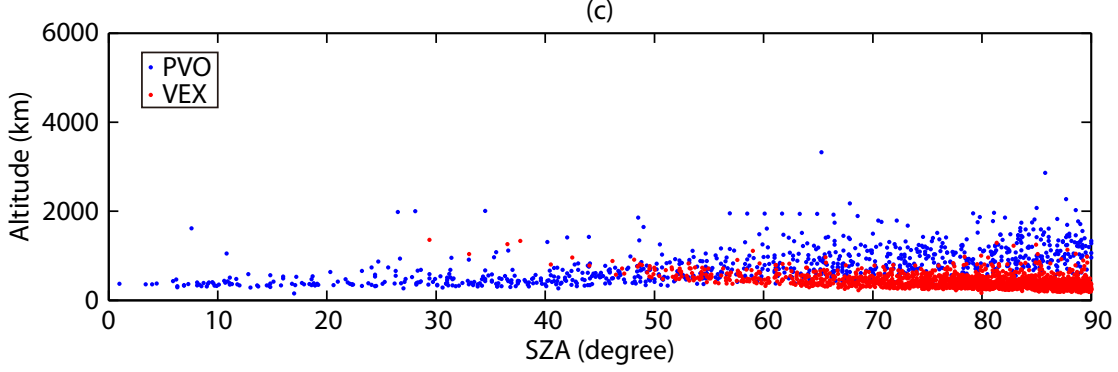

Figure 2. Distribution of the dayside ionopause data. (a) Distribution of the dayside ionopause positions measured by PVO. The color represents the altitude of the dayside ionopause in units of $\mathrm{km}$. (b) Distribution of the dayside ionopause data measured by VEX. The color represents the altitude of the dayside ionopause in units of $\mathrm{km}$. (c) Combination of PVO and VEX datasets. Blue and red dots represent the dayside ionopause altitude measured by PVO and VEX.

datasets as accurately as possible the behavior of the ionopause altitude. In this paper we display both datasets in the Venus Solar Orbital (VSO) coordinates system, where the $x$ axis points from Venus to the sun, the $y$ axis is opposite to the Venus orbital motion, and the $z$ axis is toward the ecliptic north. For simplification, we assume that the ionosphere is symmetric about the axis, and $r$ is the distance to the Venus-Sun line, $r=\sqrt{y^{2}+z^{2}}$. Since $x$ and $r$ are very much smaller compared with the distance of Venus from the Sun, SZA $\approx \arccos (x / r)$.

\subsection{Method to Reduce Orbit Bias}

We analyzed the VEX dataset and found that it includes significant orbit bias. Figure 3 shows the dayside ionopause data points (red dots) and the periapsis altitude (blue curves) of VEX. It can be seen that most of the data points are focused just above the periapsis, especially at solar minimum (2006-2010). This indicates that VEX orbits did not cover the whole range of the ionopause altitude distribution. Moreover, the data points are denser at lower altitudes than at high altitudes, which may be a consequence of the uneven sampling, i.e., VEX spent more time in low altitude regions and thus crossed the ionopause more frequently there. If we were simply to average the calculations, the resulting ionopause altitude would lower than its actual value.
In order to reduce this kind of effect, we introduce a probability $P$ based on the observation that the number of ionopause crossings $N$ in a certain region is proportional to the time the spacecraft spends in that region $T . P$ is given by the following formula:

$$
P_{i}\left(x_{i} \pm \Delta x, r_{i} \pm \Delta r\right)=\frac{N}{T} \text {. }
$$

Here, $\left(x_{i}, r_{i}\right)$ are the coordinates of the center of the grid, and $\Delta x$ and $\Delta r$ is half of the length of the grid. Then, we use these $P$ values to analyze the effects of physical factors on ionopause altitude. We group the ionopause data points according to the physical conditions; the average altitude of the ionopause in each group can be given:

$$
H=\frac{\sum_{i=1}^{n} P_{i} \times h_{i}}{\sum_{i=1}^{n} P_{i}},
$$

where $h$ is the altitude of each grid center in this group, and $n$ is the total number of grids into which the ionopause data points distribute. Here, we have grouped the data points based on the EUV range to investigate the EUV effect on ionopause altitude.

Using this method, we calculate the distribution in the grid system of $T, N$, and $P$ for both VEX and PVO observations (Figure 4 and Figure 5). From Figure $4 \mathrm{c}$ it can be seen that the most probable altitude of the ionopause (yellow curve) is at the lowest grid 


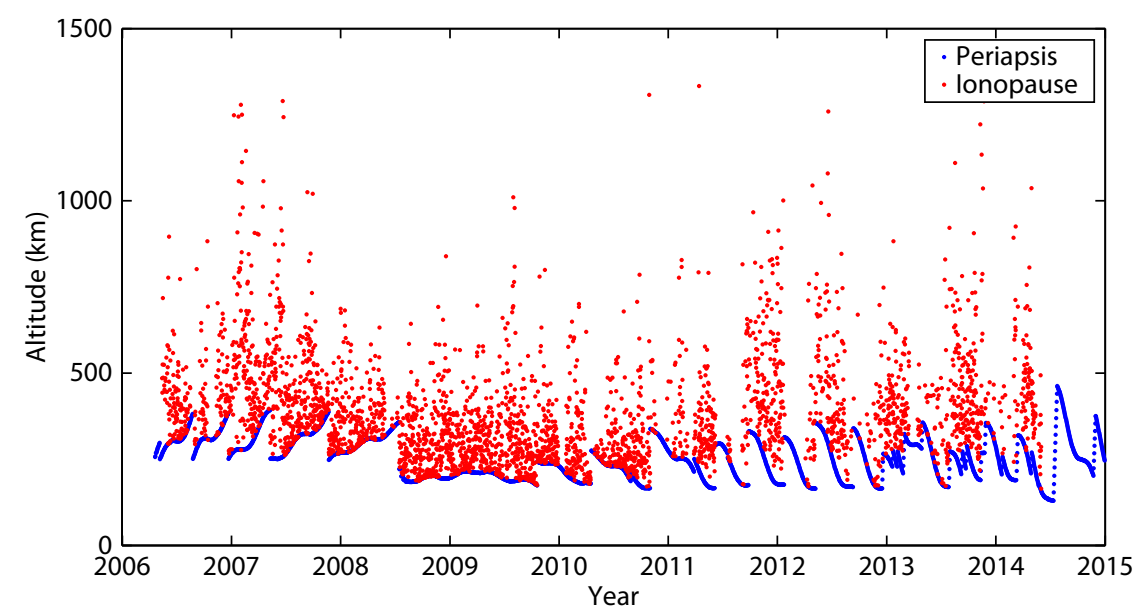

Figure 3. Distribution of VEX dayside ionopause and periapsis altitude. The red dots represent the dayside ionopause altitude and the blue curve represents the periapsis altitude. First, the ionopause data points focus just above the periapsis, especially during solar minimum. The VEX orbits did not cover the whole region where the ionopause distribute, i.e., there should be more data points below the periapsis. Second, the data points are denser in the lower altitude region which is caused by the uneven orbits distribution.

of the VEX orbit coverage, indicating that most of time the ionopause should locate at altitudes beneath VEX's periapsis. But more data points have been recorded in the lower region. The uneven sampling has made the directly calculated average ionopause altitude lower than its actual altitude. The method of using $P$ has resulted in a higher altitude, which we claim is closer to the real altitude. From Figure $5 c$ it can be seen that the PVO data points distribute both below and above the most probable altitudes (yellow curve in the bottom panel); i.e., PVO orbits cover the full range of the ionopause altitude distribution. Since the PVO spent more time in the higher region than in the lower region (Figure $5 \mathrm{a}$ ), it recorded more data points in the higher region (Figure $5 b$ ). The uneven PVO sampling has made the directly calculated average ionopause altitudes based on raw PVO data higher than its actual altitude. Using the $P$ method to normalize the data has yielded a lower altitude, which again we claim is closer to the real altitude.

\section{Results}

In order to investigate the EUV effect on the ionopause altitude over a whole solar cycle, we combined VEX and PVO datasets. Since the VEX data points are concentrated at SZA $60^{\circ}-90^{\circ}$, and the altitude did not change as much as the SZA changed, we chose from both datasets the data points in the SZA range $60^{\circ}-90^{\circ}$. Then we calculated the average altitudes $H$ of the ionopause observed in EUV flux bins, using the $P$ method for correcting orbit bias. The relationship between EUV and the dayside ionopause altitude is derived as shown by the light blue curve in Figure 6. The horizontal axis is the flux of $10.7 \mathrm{~cm}$ solar radiation, which is a proxy of solar EUV flux. The vertical axis is the altitude of the dayside ionopause. The blue points represent the selected PVO dayside ionopause data points and red points represent the VEX dayside ionopause data points. The grey solid circle represents the mean altitude before the orbit bias reduction. The light blue solid circle represents the altitude after reducing orbit bias. We find that the altitude of the dayside ionopause increases with increasing solar EUV. Fitting a cruve to the data, we derive the following equation for the dayside ionopause altitude as a function of solar EUV flux:

$$
H_{\mathrm{ip}}(\mathrm{km})=0.0065 \times F_{10.7}^{1.9239}+301.2054
$$

However, we are aware that observations are inadquate in the region below the VEX periapsis during the solar minimum. Use of the $P$ method can reduce only the orbital bias, but cannot solve the problem of lack of observations. If we consider the data points in this under-observed region, we have reason to believe that the curvature should be smaller; also needing to be taken into account is that the PEB is probably located higher than the ionospheric density fall-off (Han X et al., 2014; Han QQ et al., 2019). For these reasons, the relationship obtained here from the available observed data probably underestimates the EUV effect on the altitude of the dayside ionopause. Nevertheless, the relationship agrees with the physical theory: Since the dayside ionosphere of Venus is produced primarily by ionization of its neutral upper atmosphere due to solar EUV radiation, the EUV variation is expected to affect the production rate of ions and further to affect the morphology of the ionosphere. When the EUV solar flux increases, the production rate of the ions rises as well, thus the ionospheric thermal pressure $n k T$ is enhanced. The altitude at which the normal component of the external solar wind pressure is approximately balanced by the ionospheric pressure appears to increase.

\section{Discussion}

We have combined PVO and VEX dayside ionopause datasets to analyze the EUV effect, and have found that the dayside ionopause altitude of Venus increases with the solar EUV flux. However, due to the difference in the instruments measuring ionospheric parameters carried by PVO and VEX, different definitions of the ionopause are used to analyze the PVO and VEX observations. Here we discuss the influence on our results of the different ionopause definitions. Since MEX (Mars Express) carries the Mars Advanced Radar for Subsurface and lonospheric Sounding (MARSIS), which provides electron density profiles via local plasma oscillations, and the Analyzer of Space Plasmas and Energetic Atoms (ASPERA-3), which provides electron energy spectra, both 


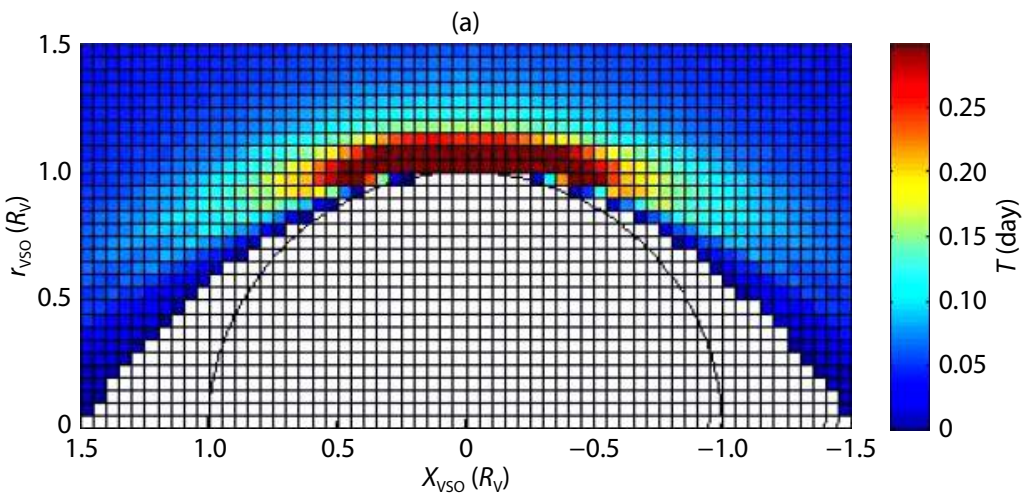

(b)

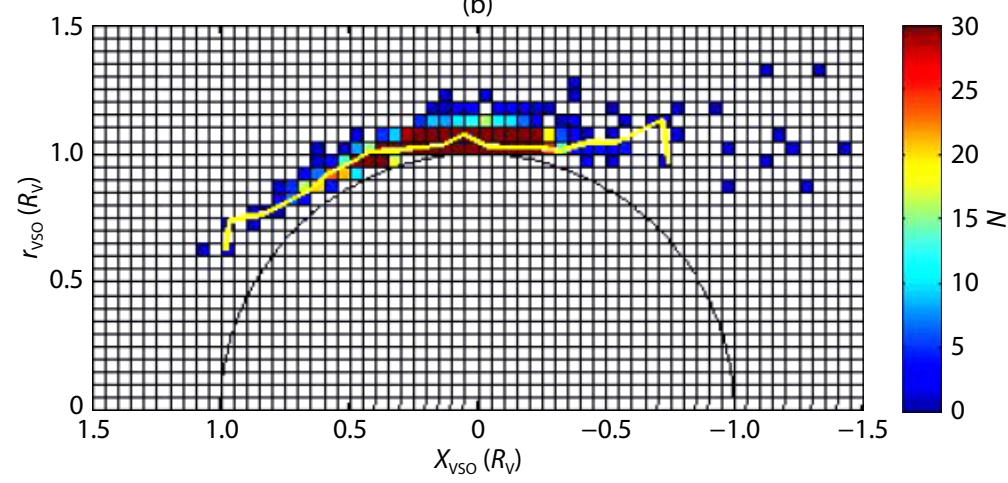

(c)

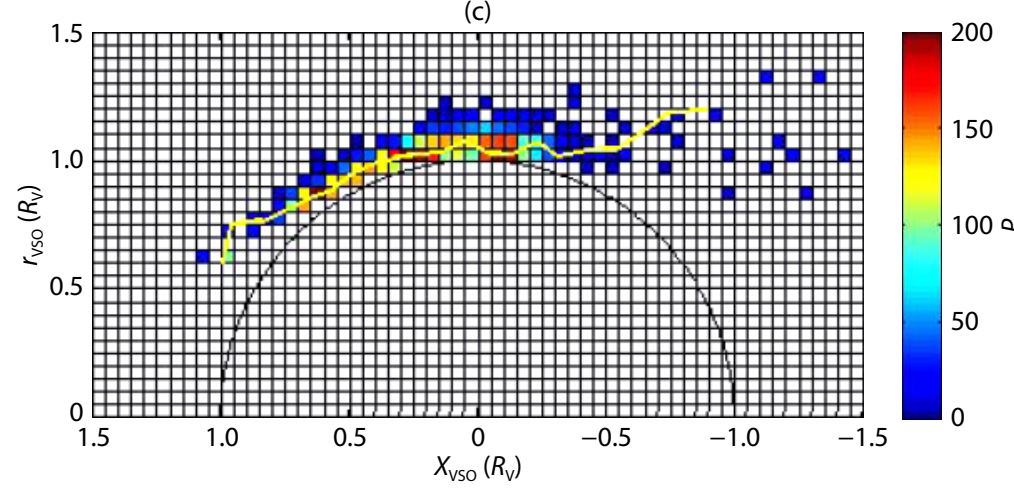

Figure 4. The sum of during period $(T)$, sum number of ionopause data points $(N)$, and probability $(P)$ distribution of the VEX ionopause observations in the grid system. $r_{\mathrm{Vso}}=\sqrt{y_{\mathrm{VSO}}^{2}+z_{\mathrm{VSO}}^{2}}$. The color from top to bottom represents the value of $T$ (day), $N$ and $P$, respectively. The yellow lines represent the position of the grid points with the largest $N$ and $P$ value as seen from the center of the coordinate system.

the ionopause and the PEB can be identified during each MEX orbit. The discrepancy between the ionopause and the PEB on Mars has been investigated by Han X et al. (2014). They defined the ionopause as the altitude at which the electron density first drops below a given value (of $10^{3} \mathrm{~cm}^{-3}$ ), and defined the PEB as the location in the ionospheric spectra at which the characteristic $\mathrm{CO}_{2}$ peaks vanish. Their approach results in an average altitude of the PEB that is about $200 \mathrm{~km}$ higher than that of the ionopause, and they suggest that the discrepancy between the ionopause and PEB is a conseqeunce of field disturbances that allow photoelectrons to travel outside. The relationship between PEB and the steep electron density gradient on Mars has been investigated also by Han QQ et al. (2019). They report that, although the PEBs do appear to be located higher than the electron density gradient boundary, on average when based on all observations, the two boundaries are nearly collocated when observed on the same orbit leg, suggesting that a possible explanation may be the magnetic field configuration in the Martian ionosphere. Considering that Venus and Mars have very similar space environments, the Venusian ionopause would be expected to be lower than its PEB, suggesting that the real curvature of the dayside ionopause altitude versus EUV should be even smaller than shown in Figure 6. Therefore, we conclude that the different definitions of ionopause altitude should not qualitatively influence our result, which is that the dayside ionopause altitude increases with the solar EUV radiation.

It can be seen that the ionopause altitudes observed by PVO decrease as SZA decrease, while those observed by VEX increase. This difference can be seen in Figure 2, Figure 4, and Figure 5. These observations are not contradictory. On one hand, the differ- 


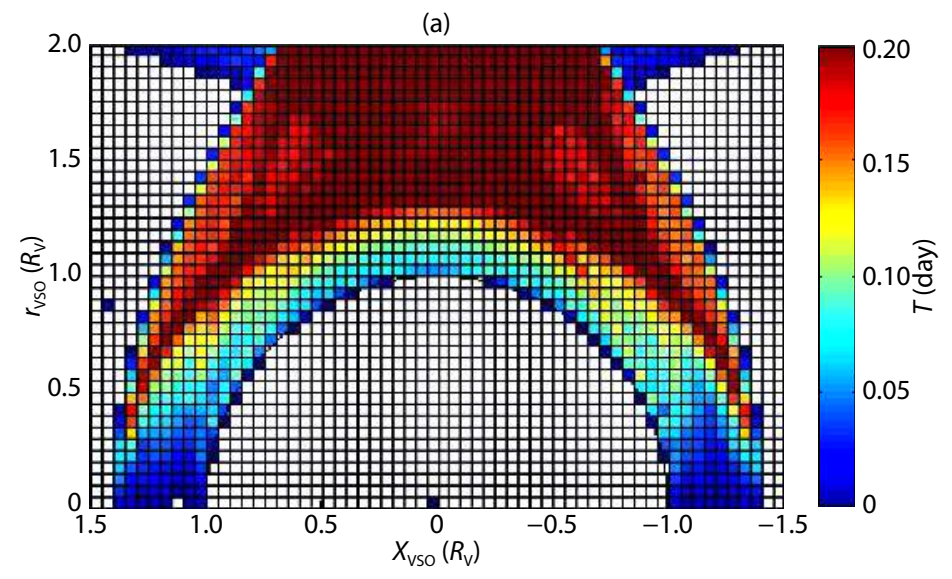

(b)
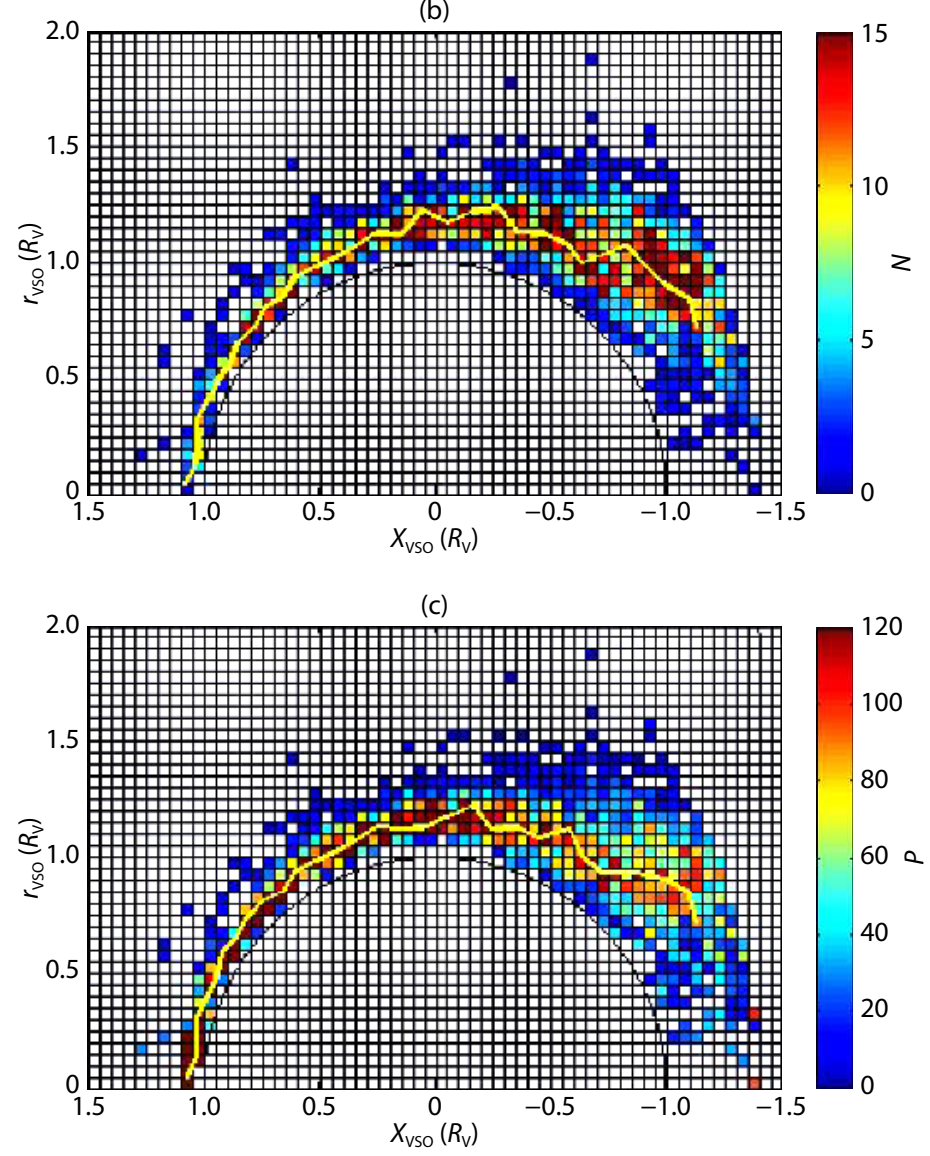

Figure 5. The sum of during period $(T)$, sum number of ionopause data points $(M)$, and probability $(P)$ distribution of the PVO ionopause observations in the grid system. $r$ Vso $=\sqrt{y_{\text {Vso }}^{2}+z_{\text {VSo }}^{2}}$. The color from top to bottom represents the value of $T$ (day), $N$ and $P$, respectively. The yellow lines represent the position of the grid points with the largest $N$ and $P$ value as seen from the center of the coordinate system.

ence is related to the sampling problem, since our $\mathrm{P}$ method does not completely eliminate the orbital biases. On the other hand, the electron density profiles measured by the Venara 9 and 10 radio occulation experiment near the minimum of sunspot cycle 21 show that near the terminator the ionopause altitude decreases as the SZA increases (Mahajan et al., 1989). Most of our knowledge about the Venusian ionopause comes from Pioneer Venus Orbiter (PVO) observations taken during a solar maximum. When solar wind pressure is high (or the ionosphere is weak, as is the case during solar minimum), the process responsible for forma- tion of the ionopause is entirely different from that when solar wind pressure is low (during solar maximum).

When $P_{\text {sw }}$ is high, the diffusion region is compressed, and the concentration of the neutral atmosphere is now sufficiently large that the ionization produced by the absorbtion of EUV becomes important. Photoionization replenishes the plasma that is carried away by the solar wind, causing the plasma to be distributed with the scale height of atomic oxygen. Since the scale height of atomic oxygen is relatively low, one observes only small changes in the ionopause altitude as the solar wind pressure changes. The ab- 


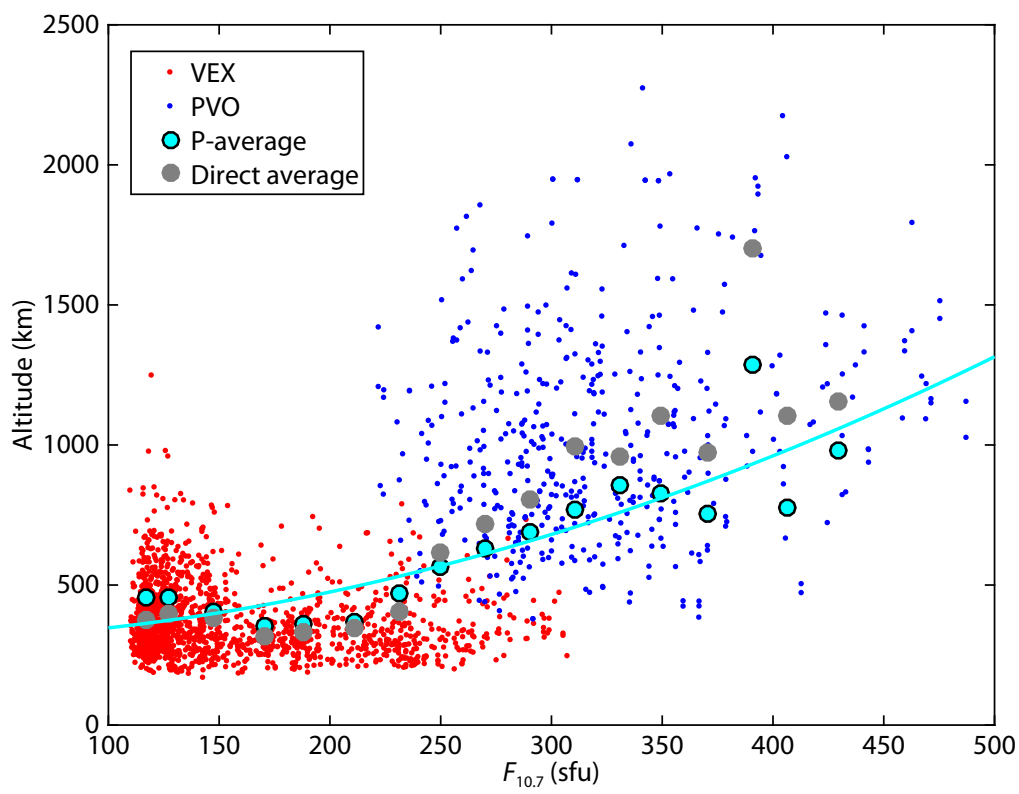

Figure 6. Plot of observed ionopause altitude vs. solar $F_{10.7}$ flux in sfu. The data were averaged in 20 sfu bins in $F_{10.7}$ and also over plotted in light blue solid dots by reducing the orbit bias. The light blue dots represent the data averaged in 20 sfu with the P method. The grey dots represent the data averaged in 20 sfu bins without reducing the orbit bias. From the statistical fit to the data, we get the relationship between the dayside ionopause altitude and solar EUV flux shown by the light blue curve.

sorption of EUV decreases as SZA increases; thus the VEX measurements of ionopause altitude decrease. This ionopause is called the photodynamical ionopause and has been investigated based on the radio occultation experiment during solar minimum (Mahajan et al., 1989; Mahajan and Mayr, 1989). Since the Mars and Venus ionospheres are similar during high solar wind conditions, understanding the Mars ionopause is relevant to the Venus case.

It is especially useful to compare the EUV-dependence of the ionopause of Venus to that of Mars. Sánchez-Cano et al. (2015) investigated the solar cycle effect on the topside ionosphere of Mars. They analyzed the variation of the topside ionosphere electron density within an entire solar cycle. They reported that the topside ionospheric density in the period of extremely low solar activity was smaller than in other phases of the solar cycle. This agrees with the result of our present study, that the altitude of the dayside ionopause increases with the solar EUV flux. However, in their study, the ionospheric density at extremely low solar activity did not show a dependence on EUV flux. On the other hand, Edberg et al. (2009) analyzed magnetic pileup boundary (MPB) crossings observed by MEX from February 2004 to January 2009, and found that the MPB decreases linearly with increasing EUV flux during the declining phase of the solar cycle and during solar minimum. These results suggest that solar wind interaction with an unmagnetized body is complicated. In the near future, the influence of magnetic fields on the morphology of ionopause during one solar cycle should be investigated on both sides of the ionopause, to reveal more details of the variablity of ionospheres with solar activity.

It is worthy of note that, compared with VEX dayside ionopause positions, PVO dayside ionopause positions are wider spread in altitude, as shown in Figure 6. This difference is probably associated with variation in solar wind dynamic pressure. PVO dayside ionopause data used in the present study were collected at solar maximum whereas VEX data were collected during almost one entire solar cycle. The solar wind dynamic pressure is more variable in solar maximum than at other times in the cycle, especially than at solar minimum. The location of the ionopause depends on the solar wind dynamic pressure and ionospheric pressure (Luhmann and Cravens, 1991). The ionopause is controlled by pressure balance, thus the altitude of the dayside ionopause is sensitive to solar wind variations. When the solar wind dynamic pressure is low, purely thermal ionospheric pressure can balance the normal solar wind dynamic pressure, so the ionopause forms at relatively high altitude. VEX observations demonstrate that the ionosphere can significantly expand during an extremely low solar wind dynamic pressure event (Wei Y et al., 2012). If the solar wind dynamic pressure is very high, the ionospheric thermal pressure itself is insufficient to balance the solar wind dynamic pressure. In this case, the interplanetary magnetic field (IMF) can permeate into the ionosphere, adding magnetic pressure to plasma thermal pressure in the ionosphere to balance the solar wind dynamic pressure. The ionopause may then contract and reach a lower altitude. However, when the external pressure exceeds $4 \times 10^{8}$ dyne $\mathrm{cm}^{-2}$, the altitude of the ionopause reaches a lower limit, and it decreases only a little when the external pressure increases. Mahajan and Mayr (1989) have depicted this saturation phenomenon, which occurs when the ionopause is driven deeply into the thermosphere.

\section{Conclusion}

We have combined PVO and VEX datasets to analyze the EUV-dependence of the Venusian dayside ionopause altitude, and have found that the dayside ionopause altitude of Venus increases when the solar EUV flux increases. Our approach has yielded a 
curve with a larger curvature than previously reported, because the VEX database does not contain ionopause records below its periapsis. We suggest that a future Venus mission should consider a global survey of the ionosphere, especially the lower part of the ionosphere during solar minimum.

\section{Acknowledgments}

The PVO data are available at the Planetary Data System https://pds-ppi.igpp.ucla.edu/). The VEX ELS data are available at the ESA Planetary Science Archive (http://www.cosmos.esa.int/). The $F_{10.7}$ solar flux data came from http://www.mikechaffin.net/ research/solar-flux-proxies-for-earth-mars-and-venus/. The work was supported by the National Natural Science Foundation of China $(41525016,41525015,41661164034,41621063)$ and the National Important Basic Research Project (2011CB811405). Y. Wei is supported by the Thousand Young Talents Program of China. We acknowledge the role of ISSI and ISSI-BJ in supporting the science discussions within International Team 317 that contributed to this study.

\section{References}

Angsmann, A., Fränz, M., Dubinin, E., Woch, J., Barabash, S., Zhang, T. L., and Motschmann, U. (2011). Magnetic states of the ionosphere of Venus observed by Venus Express. Planet. Space Sci., 59(4), 327-337. https://doi.org/10.1016/j.pss.2010.12.004

Barabash, S., Sauvaud, J. A., Gunell, H., Andersson, H., Grigoriev, A., Brinkfeldt, K., Holmström, M., Lundin, R., Yamauchi, M., ... Bochsler, P. (2007). The analyser of space plasmas and energetic atoms (ASPERA-4) for the venus express mission. Planet. Space Sci., 55(12), 1772-1792. https://doi.org/10.1016/j.pss.2007.01.014

Brace, L. H., Theis, R. F., Hoegy, W. R., Wolfe, J. H., Mihalov, J. D., Russell, C. T., Elphic, R. C., and Nagy, A. F. (1980). The dynamic behavior of the Venus ionosphere in response to solar wind interactions. J. Geophys. Res., 85(A13), 7663-7678. https://doi.org/10.1029/JA085iA13p07663

Brace, L. H., Hoegy, W. R., and Theis, R. F. (1988). Solar EUV measurements at Venus based on photoelectron emission from the Pioneer Venus Langmuir Probe. J. Geophys. Res., 93(A7), 7282-7296. https://doi.org/10.1029/JA093iA07p07282

Brace, L. H., and Kliore, A. J. (1991). The structure of the Venus ionosphere. Space Sci. Rev., 55(1-4), 81-163. https://doi.org/10.1007/BF00177136

Coates, A. J., Frahm, R. A., Linder, D. R., Kataria, D. O., Soobiah, Y., Collinson, G., Sharber, J, R., Winningham, J. D., Jeffers, S. J., ... Grande, M. (2008). Ionospheric photoelectrons at Venus: Initial observations by ASPERA-4 ELS. Planet. Space Sci., 56(6), 802-806. https://doi.org/10.1016/j.pss.2007.12.008

Edberg, N. J. T., Brain, D. A., Lester, M., Cowley, S. W. H., Modolo, R., Fränz, M., and Barabash, S. (2009). Plasma boundary variability at Mars as observed by Mars Global Surveyor and Mars Express. Ann. Geophys., 27(9), 3537-3550. https://doi.org/10.5194/angeo-27-3537-2009

Fox, J. L., and Kliore, A. J. (1997). lonosphere: solar cycle variations. In S. W. Bougher, et al. (Eds.), Venus II: Geology, Geophysics, Atmosphere, and Solar Wind Environment. Tucson, AZ: University of Arizona Press.

Han, Q. Q., Fan, K., Cui, J., Wei, Y., Fraenz, M., Dubinin, E., Chai, L. H., Rong, Z. J.,
Wan, W. X., ... Connerney, J. E. P. (2019). The relationship between photoelectron boundary and steep electron density gradient on Mars: MAVEN observations. J. Geophys. Res., 124(10), 8015-8022. https://doi.org/10.1029/2019JA026739

Han, X., Fraenz, M., Dubinin, E., Wei, Y., Andrews, D. J., Wan, W., He, M., Rong, Z. J., Chai, L., ... Barabash, S. (2014). Discrepancy between ionopause and photoelectron boundary determined from Mars Express measurements. Geophys. Res. Lett., 41(23), 8221-8227. https://doi.org/10.1002/2014GL062287

Krehbiel J. P., Brace, L. H., Theis, R. F., Cutler, J. R., Pinkus, W. H., and Kaplan, R. B. (1980). Pioneer Venus Orbiter electron temperature probe. IEEE Trans. Geosci. Remote Sens, GE-18(1), 49-54. https://doi.org/10.1109/TGRS.1980.350260

Luhmann, J. G. (1986). The solar wind interaction with Venus. Space Sci. Rev., 44(3-4), 241-306. https://doi.org/10.1007/BF00200818

Luhmann, J. G., and Cravens, T. E., (1991). Magnetic fields in the ionosphere of Venus. Space Sci. Rev., 55(1-4), 201-274. https://doi.org/10.1007/BF00177138

Mahajan, K. K., Mayr, H. G., Brace, L. H., and Cloutier, P. A. (1989). On the lower altitude limit of the Venusian ionopause. Geophys. Res. Lett., 16(7), 759-762. https://doi.org/10.1029/GL016i007p00759

Mahajan, K. K., and Mayr, H. G. (1989). Venus lonopause during solar miminum. Geophys. Res. Lett., 16(12), 1477-1480. https://doi.org/10.1029/GL016i012p01477

Martinecz, C., Fränz, M., Woch, J., Krupp, N., Roussos, E., Dubinin, E., Motschmann, U., Barabash, S., Lundin, R., ... Lammer, H. (2008). Location of the bow shock and ion composition boundaries at Venus-initial determinations from Venus Express ASPERA-4. Planet. Space Sci., 56(6), 780-784. https://doi.org/10.1016/j.pss.2007.07.007

Martinecz, C., Boesswetter, A., Fränz, M., Roussos, E., Woch, J., Krupp, N., Dubinin, E., Motschmann, U., Wiehle, S., ... Kulikov, Y. (2009). Plasma environment of Venus: comparison of Venus Express ASPERA-4 measurements with 3-D hybrid simulations. J. Geophys. Res., 114(E9), E00B30. https://doi.org/10.1029/2008JE003174

Mitchell, D. L., Lin, R. P., Mazelle, C., Rème, H., Cloutier, P. A., Connerney, J. E. P., Acuña, M. H., and Ness, N. F. (2001). Probing Mars' crustal magnetic field and ionosphere with the MGS electron reflectometer. J. Geophys. Res., 106(E10), 23419-23427. https://doi.org/10.1029/2000JE001435

Phillips, J. L., Luhmann, J. G., Knudsen, W. C., and Brace, L. H. (1988). Asymmetries in the location of the Venus lonopause. J. Geophys. Res., 93(A5), 3927-3941. https://doi.org/10.1029/JA093iA05p03927

Russell, C. T., Zhang, T. L., and Luhmann, J. G. (1993). On the cause of distant bow shock encounters. In T. I. Gombosi (Ed.), Plasma Environments of NonMagnetic Planets (pp. 241-246). Oxford: Pergamon.

Sánchez-Cano, B., Lester, M., Witasse, O., Milan, S. E., Hall, B. E. S., Blelly, P. L., Radicella, S. M., and Morgan, D. D. (2015). Evidence of scale height variations in the Martian ionosphere over the solar cycle. J. Geophys. Res., 120(12), 10913-10925. https://doi.org/10.1002/2015JA021949

Vech, D., Szego, K., Opitz, A., Kajdic, P., Fraenz, M., Kallio, E., and Alho, M. (2015). Space weather effects on the bow shock, the magnetic barrier, and the ion composition boundary at Venus. J. Geophys. Res., 120(6), 4613-4627. https://doi.org/10.1002/2014JA020782

Wei, Y., Fraenz, M., Dubinin, E., Coates, A. J., Zhang, T. L., Wan, W., Feng, L., Angsmann, A., Opitz, A., ... Lundin, R. (2012). A teardrop-shaped ionosphere at Venus in tenuous solar wind. Planet. Space Sci., 73(1), 254-261. https://doi.org/10.1016/j.pss.2012.08.024 